Modeling, Identification and Control, Vol. 32, No. 2, 2011, pp. 47-55, ISSN 1890-1328

\title{
Mean-Square Filtering for Polynomial System States Confused with Poisson Noises over Polynomial Observations
}

\author{
Michael Basin ${ }^{1}$ Juan J. Maldonado ${ }^{1}$ Hamid Reza Karimi ${ }^{2}$
}

\begin{abstract}
${ }^{1}$ Department of Physical and Mathematical Sciences, Autonomous University of Nuevo Leon, Apdo postal 144-F, C.P. 66450, San Nicolas de los Garza, Nuevo Leon, Mexico, Phone: + 5281 83294030, Fax: + 52 81 83522954, E-mail: mbasin@fcfm.uanl.mx; jjmo.uan/@gmail.com

${ }^{2}$ Department of Engineering, Faculty of Engineering and Science, University of Agder, Grimstad, N-4898, Norway, Phone: +473723 3259, Fax: +473723 3001, E-mail: hamid.r.karimi@uia.no
\end{abstract}

\begin{abstract}
In this paper, the mean-square filtering problem for polynomial system states confused with white Poisson noises over polynomial observations is studied proceeding from the general expression for the stochastic Ito differentials of the mean-square estimate and the error variance. In contrast to the previously obtained results, the paper deals with the general case of nonlinear polynomial states and observations with white Poisson noises. As a result, the Ito differentials for the mean-square estimate and error variance corresponding to the stated filtering problem are first derived. The procedure for obtaining an approximate closed-form finite-dimensional system of the filtering equations for any polynomial state over observations with any polynomial drift is then established. In the example, the obtained closed-form filter is applied to solve the third order sensor filtering problem for a quadratic state, assuming a conditionally Poisson initial condition for the extended third order state vector. The simulation results show that the designed filter yields a reliable and rapidly converging estimate.
\end{abstract}

Keywords: Filter Design; Poisson Noises; Polynomial Observations

\section{Introduction}

Although the general solution of the mean-square filtering problem for nonlinear state and observation equations confused with white Gaussian noises is given by the Kushner equation for the conditional density of an unobserved state with respect to observations Kushner (1964), there are a very few known examples of nonlinear systems where the Kushner equation can be reduced to a finite-dimensional closed system of filtering equations for a certain number of lower conditional moments (see Kalman and Bucy (1961), Wonham (1965) and Benes (1981) for more details). The complete classification of the "general situation" cases (this means that there are no special assumptions on the structure of state and observation equations and the initial conditions), where the nonlinear finite-dimensional filter exists, is given in Yau (1994). There also exists an extensive bibliography on robust, in particular, $H_{\infty}$ filtering for linear (Xu and Chen (2003), Mahmoud and Shi (2003) and Xu et al. (2005)) and nonlinear (Xie et al. (1996), Nguang and Fu (1996), Fridman and Shaked (1997), Shi (1998),Fleming and McEneaney (2001), Yaz and Yaz (2001),Xu and van Dooren (2002),Wang et al. (2003), Gao and Wang (2004),Zhang et al. (2005), Gao et al. (2005), Zhang et al. (2007), Gao and Chen (2007), Wang et al. (2008), Wang et al. (2009),Wei et al. (2009) and Shen et al. (2009)) stochas- 
tic systems. Apart form the "general situation," the mean-square finite-dimensional filters have been designed for certain classes of polynomial system states with Gaussian noises over linear observations (Basin (2008), Basin et al. (2008) and Basin et al. (2009)) and a few results related to nonlinear Poisson systems can be found in Lu et al. (2001), Kolmanovsky and Maizenberg (2002a),Hannequin and Mas (2002),Kolmanovsky and Maizenberg (2002b),Zhang et al. (2008a),Dupé et al. (2008),Zhang et al. (2008b), and Basin and Maldonado (2011). Recently, the mean-square filtering problem for polynomial systems, where both, state and observation, equations include polynomial functions of the system state in the right-hand sides, was solved in Basin et al. (2010); however, that paper did not consider systems corrupted with non-Gaussian noises. On the other hand, it is well-known that there are a number of practical situations where dynamic system states are corrupted not with uniformly acting white Gaussian noises (like a static noise in a phone line) but with noises acting at random isolated time moments (like a series of electromagnetic impulses), which are referred to as white Poisson noises.

This paper presents an approximate finitedimensional filter for polynomial system states confused with white Poisson noises over polynomial observations, continuing the research in the area of the mean-square filtering for polynomial systems with Gaussian (Basin (2008), Basin et al. (2008) and Basin et al. (2009)) and Poisson Basin and Maldonado (2011) noises. In contrast to the previously obtained results, the paper deals with the general case of nonlinear polynomial states and observations with white Poisson noises. Designing a closed-form finite-dimensional filter for systems with white Poisson noises over polynomial observations presents a significant advantage in the filtering theory and practice, since it enables one to address some filtering problems with state and observation nonlinearities and non-Gaussian noises, such as the cubic sensor problem Hazewinkel et al. (1983), for various polynomial systems. Indeed, the main paper result allows one to design a suboptimal mean-square finite-dimensional filter for any polynomial state confused with white Poisson noises over polynomial observations. Furthermore, since any nonlinear function can be approximated by a polynomial of a certain degree up to any precision, this would potentially lead to designing a suboptimal mean-square finite-dimensional filter for any nonlinear state with Poisson noises over observations with a nonlinear drift. The stated filtering problem is treated proceeding from the general expression for the stochastic Ito differentials of the mean-square estimate and the error variance Pugachev and Sinitsyn (2001).
As the first result, the Ito differentials for the meansquare estimate and error variance corresponding to the stated filtering problem are derived. Then, a closed-form finite-dimensional system of the filtering equations with respect to a finite number of filtering variables can be obtained for a polynomial observation equation, additionally assuming a conditionally Poisson initial condition for the higher degree states. This assumption is quite admissible in the filtering framework, since the real distribution of the entire state vector is actually unknown. In this case, the corresponding procedure for designing the closed-form filtering equations is suggested.

As an illustrative example, the closed system of the filtering equations with respect to two variables, the mean-square estimate and the error variance, is derived in the explicit form for the particular case of a quadratic state and third order polynomial observations, assuming a conditionally Poisson initial condition for the extended third order state vector. This filtering problem generalizes the cubic sensor problem stated in Hazewinkel et al. (1983). The resulting filter yields a reliable and rapidly converging estimate, in spite of a significant difference in the initial conditions between the state and estimate, whereas the filter designed for systems with white Gaussian noises, constructed according to Basin et al. (2009) and Basin and Maldonado (2011), behaves unsatisfactorily.

The paper is organized as follows. Section 2 presents the filtering problem statement for polynomial system states confused with white Poisson noises over polynomial observations. The Ito differentials for the meansquare estimate and the error variance are derived in Section 3, where the procedure for obtaining an approximate finite-dimensional filter is suggested for any polynomial state with Poisson noises over observations with any polynomial drift. In Section 4, the obtained filter is applied to solution of the third order sensor filtering problem for a quadratic state, assuming a conditionally Poisson initial condition for the extended third order state vector.

Notation. The following notation is accepted throughout the paper: for vectors $z \in R^{m}$ and $x \in R^{n}$, $[z, x] \in R^{m+n}$ denotes a column vector consecutively composed of $m$ components of the vector $z$ and $n$ components of the vector $x$ in the same order; $[I, 0]$ denotes the $m \times(n+m)$ matrix consecutively composed of the $m \times m$-dimensional identity matrix and $m \times n$ dimensional zero matrix.

\section{Problem Statement}

Let $(\Omega, F, P)$ be a complete probability space with an increasing right-continuous family of $\sigma$-algebras $F_{t}, t \geq$ 
$t_{0}$, and let $\left(N_{1}(t), F_{t}, t \geq t_{0}\right)$ and $\left(N_{2}(t), F_{t}, t \geq t_{0}\right)$ be independent Poisson processes. The $F_{t}$-measurable random process $(x(t), y(t))$ is described by nonlinear polynomial differential equations for the system state

$$
d x(t)=\rho(x, t) d t+\sigma(x, t) d N_{1}(t), \quad x\left(t_{0}\right)=x_{0},
$$

and the observation process

$$
d y(t)=h(x, t) d t+B(t) d N_{2}(t) .
$$

Here, $x(t) \in R^{n}$ is the state vector and $y(t) \in R^{m}$ is the observation vector. The initial condition $x_{0} \in R^{n}$ is a Poisson vector such that $x_{0}, N_{1}(t) \in R^{p}$, and $N_{2}(t) \in$ $R^{q}$ are independent. It is assumed that $B(t) B^{T}(t)$ is a positive definite matrix, therefore, $m \leq q$. All coefficients in (1)-(2) are deterministic functions of appropriate dimensions. Solutions of the Ito stochastic differential equations (1) and (2) are regarded as weak solutions (see, for example, Oksendal (2006) for definition). The same definition holds for solutions of other Ito stochastic differential equations throughout the paper.

The nonlinear functions $\rho(x, t) \in R^{n}, \sigma(x, t) \in R^{n}$, and $h(x, t) \in R^{m}$ are considered polynomials of $n$ variables, components of the state vector $x(t) \in R^{n}$, with time-dependent coefficients. Since $x(t) \in R^{n}$ is a vector, this requires a special definition of the polynomial for $n>1$. In accordance with Basin (2008), a $p$-degree polynomial of a vector $x(t) \in R^{n}$ is regarded as a $p$ linear form of $n$ components of $x(t)$

$$
\begin{aligned}
\rho(x, t)= & \alpha_{0}(t)+\alpha_{1}(t) x+\alpha_{2}(t) x x^{T}+\ldots \\
& +\alpha_{p}(t) x \ldots p \text { times } \ldots x,
\end{aligned}
$$

where $\alpha_{0}(t)$ is a vector of dimension $n, \alpha_{1}$ is a matrix of dimension $n \times n, \alpha_{2}$ is a $3 \mathrm{D}$ tensor of dimension $n \times n \times n, \alpha_{p}$ is an $(p+1) \mathrm{D}$ tensor of dimension $n \times$ $\cdots(p+1)$ times $\cdots \times n$, and $x \times \ldots p$ times $\ldots \times x$ is a $p \mathrm{D}$ tensor of dimension $n \times \ldots p$ times $\ldots \times n$ obtained by $p$ times spatial multiplication of the vector $x(t)$ by itself (see Basin (2008) for more details). Such a polynomial can also be expressed in the summation form

$$
\begin{gathered}
\rho_{k}(x, t)=\alpha_{0 k}(t)+\sum_{i} \alpha_{1 k i}(t) x_{i}(t) \\
+\sum_{i j} \alpha_{2 k i j}(t) x_{i}(t) x_{j}(t)+\ldots \\
+\sum_{i_{1} \ldots i_{p}} \alpha_{p k i_{1} \ldots i_{p}}(t) x_{i_{1}}(t) \ldots x_{i_{p}}(t), \\
k, i, j, i_{1}, \ldots, i_{p}=1, \ldots, n .
\end{gathered}
$$

The estimation problem is to find the mean-square estimate $\hat{x}(t)$ of the system state $x(t)$, based on the observation process $Y(t)=\{y(s), 0 \leq s \leq t\}$, that minimizes the conditional expectation of the Euclidean norm

$$
J=E\left[(x(t)-\hat{x}(t))^{T}(x(t)-\hat{x}(t)) \mid F_{t}^{Y}\right]
$$

at every time moment $t$. Here, $E\left[\xi(t) \mid F_{t}^{Y}\right]$ means the conditional expectation of a stochastic process $\xi(t)=(x(t)-\hat{x}(t))^{T}(x(t)-\hat{x}(t))$ with respect to the $\sigma$ - algebra $F_{t}^{Y}$ generated by the observation process $Y(t)$ in the interval $\left[t_{0}, t\right]$. As known Pugachev and Sinitsyn (2001), the mean-square estimate is given by the conditional expectation

$$
\hat{x}(t)=m_{x}(t)=E\left(x(t) \mid F_{t}^{Y}\right)
$$

of the system state $x(t)$ with respect to the $\sigma$ - algebra $F_{t}^{Y}$ generated by the observation process $Y(t)$ in the interval $\left[t_{0}, t\right]$. As usual, the matrix function

$$
P(t)=E\left[\left(x(t)-m_{x}(t)\right)\left(x(t)-m_{x}(t)\right)^{T} \mid F_{t}^{Y}\right]
$$

is the estimation error variance matrix. Hereinafter, the formulated filtering problem is considered in a time interval $\left[t_{0}, T_{1}\right]$, where the solution of the state equation (1) still exists and is almost surely bounded. Apparently, $T_{1}<T^{*}$, where $T^{*}$ is an escape time for the system (1). Note that since the initial condition $x_{0}$ is Poisson, the superior moments of the process $x(t)$ remain bounded for any $t \leq T_{1}$ (Pugachev and Sinitsyn (2001)).

The proposed solution to the stated filtering problem is based on the formulas for the Ito differentials of the mean-square estimate and the estimation error variance (cited after Pugachev and Sinitsyn (2001)) and given in the following section.

\section{Filter Design}

The filtering problem is solved by the following theorem.

Theorem 1. The mean-square filter for the polynomial state $x(t)$ (1) over the polynomial observations $y(t)$ (2) is given by the following equations for the mean-square estimate $m(t)=\left[m_{z}(t), m_{x}(t)\right]=$ $E\left([z(t), x(t)] \mid F_{t}^{Y}\right)$ and the estimation error covariance matrix $P(t)=E[([z(t), x(t)]-m(t))([z(t), x(t)]-$ $\left.m(t))^{T} \mid F_{t}^{Y}\right]$ :

$$
\begin{gathered}
d m(t)=E\left(\bar{f}(x, t) \mid F_{t}^{Y}\right) d t \\
+P(t)[I, 0]^{T}\left(B(t) B^{T}(t)\right)^{-1}\left(d y(t)-m_{z}(t) d t\right), \\
d P(t)=\left(E\left(([z(t), x(t)]-m(t))(\bar{f}(x, t))^{T} \mid F_{t}^{Y}\right)\right. \\
\left.+E\left(\bar{f}(x, t)([z(t), x(t)]-m(t))^{T}\right) \mid F_{t}^{Y}\right)+ \\
E\left(\bar{g}(x, t) \bar{g}^{T}(x, t) \mid F_{t}^{Y}\right)
\end{gathered}
$$




$$
\left.-P(t)[I, 0]^{T}\left(B(t) B^{T}(t)\right)^{-1}[I, 0] P(t)\right),
$$

with the initial conditions $m\left(t_{0}\right)=\left[m_{z}\left(t_{0}\right), m_{x}\left(t_{0}\right)\right]=$ $E\left(\left[z_{0}, x_{0}\right] \quad \mid \quad F_{t_{0}}^{Y}\right)$ and $P\left(t_{0}\right)=E\left[\left(\left[z_{0}, x_{0}\right]-\right.\right.$ $m\left(t_{0}\right)\left(\left[z_{0}, x_{0}\right]-m\left(t_{0}\right)^{T} \mid F_{t_{0}}^{Y}\right]$. Here, $\bar{f}(x, t)=$ $[f(x, t), \rho(x, t)], \bar{g}(x, t)=[g(x, t), \sigma(x, t)]^{T}$,

$$
f(x, t)=\frac{\partial h(x, t)}{\partial x} \rho(x, t) d t+\frac{\partial h(x, t)}{\partial t} d t+
$$

$\frac{1}{2} \frac{\partial^{2} h(x, t)}{\partial x^{2}} \sigma(x, t) \sigma^{T}(x, t) d t, \quad g(x, t)=\frac{\partial h(x, t)}{\partial x} \sigma(x, t)$, and the additional polynomial state $z(t)=h(x, t)$ satisfies the equation

$$
\begin{gathered}
d z(t)=\frac{\partial h(x, t)}{\partial x} \rho(x, t) d t+\frac{\partial h(x, t)}{\partial t} d t+ \\
\frac{1}{2} \frac{\partial^{2} h(x, t)}{\partial x^{2}} \sigma(x, t) \sigma^{T}(x, t) d t+ \\
\frac{\partial h(x, t)}{\partial x} \sigma(x, t) d N_{1}(t), \quad z(0)=z_{0} .
\end{gathered}
$$

If the initial condition $\left[z_{0}, x_{0}\right]$ for the extended state vector is conditionally Poisson with respect to the observations, the system of filtering equations (4),(5) becomes a closed-form finite-dimensional system after expressing the superior conditional moments of the system state $x(t)$ with respect to the observations $y(t)$ as functions of only two lower conditional moments, $m(t)$ and $P(t)$.

Proof. Let us reformulate the problem, introducing the stochastic process $z(t)=h(x, t)$. Using the Ito formula (see Pugachev and Sinitsyn (2001)) for the stochastic differential of the nonlinear function $h(x, t)$, where $x(t)$ satisfies the equation (1), the equation (6) is obtained for $z(t)$

$$
\begin{gathered}
d z(t)=\frac{\partial h(x, t)}{\partial x} \rho(x, t) d t+\frac{\partial h(x, t)}{\partial t} d t+ \\
\frac{1}{2} \frac{\partial^{2} h(x, t)}{\partial x^{2}} \sigma(x, t) \sigma^{T}(x, t) d t+ \\
\frac{\partial h(x, t)}{\partial x} \sigma(x, t) d N_{1}(t), \quad z(0)=z_{0} .
\end{gathered}
$$

Note that the addition $\frac{1}{2} \frac{\partial^{2} h(x, t)}{\partial x^{2}} \sigma(x, t) \sigma^{T}(x, t)$ appears in view of the second derivative in $x$ in the Ito formula.

Let us assume at this point that the initial condition $\left[z_{0}, x_{0}\right]$ for the extended state vector is a conditionally Poisson random vector with respect to observations. This assumption is quite admissible in the filtering framework, since the real distributions of $x(t)$ and $z(t)$ are actually unknown. Indeed, as follows from $\mathrm{Pu}-$ gachev (1984), if only two lower conditional moments, expectation $m_{0}$ and variance $P_{0}$, of a random vector $\left[z_{0}, x_{0}\right]$ are available, the Poisson distribution with the same parameters, $m_{0}$ and $P_{0}$, is the best approximation for the unknown conditional distribution of $\left[z_{0}, x_{0}\right]$ with respect to observations. This fact is also a corollary of the central limit theorem Tucker (1967) in the probability theory.

A key point for further derivations is that the righthand side of the equation (6) is a polynomial in $x$. Indeed, since $h(x, t)$ is a polynomial in $x$, the functions $\frac{\partial h(x, t)}{\partial x}, \frac{\partial h(x, t)}{\partial x} x(t), \frac{\partial h(x, t)}{\partial t}$, and $\frac{\partial^{2} h(x, t)}{\partial x^{2}}$ are also polynomial in $x$. Thus, the equation (6) is a polynomial state equation with a polynomial multiplicative noise. It can be written in the compact form

$$
d z(t)=f(x, t) d t+g(x, t) d N_{1}(t), \quad z\left(t_{0}\right)=z_{0},
$$

where

$$
\begin{gathered}
f(x, t)=\frac{\partial h(x, t)}{\partial x} \rho(x, t) d t+\frac{\partial h(x, t)}{\partial t} d t+ \\
\frac{1}{2} \frac{\partial^{2} h(x, t)}{\partial x^{2}} \sigma(x, t) \sigma^{T}(x, t) d t, \quad g(x, t)=\frac{\partial h(x, t)}{\partial x} \sigma(x, t) .
\end{gathered}
$$

In terms of the process $z(t)$, the observation equation (2) takes the form

$$
d y(t)=[I, 0][z(t), x(t)] d t+B(t) d N_{2}(t) .
$$

The reformulated estimation problem is now to find the mean-square estimate $\left[m_{z}(t), m_{x}(t)\right]$ of the system state $[z(t), x(t)]$, based on the observation process $Y(t)=\{y(s), 0 \leq s \leq t\}$. This mean-square estimate is given by the conditional expectation

$$
m(t)=\left[m_{z}(t), m_{x}(t)\right]=\left[E\left(z(t) \mid F_{t}^{Y}\right), E\left(x(t) \mid F_{t}^{Y}\right)\right]
$$

of the system state $[z(t), x(t)]$ with respect to the $\sigma$ algebra $F_{t}^{Y}$ generated by the observation process $Y(t)$ in the interval $\left[t_{0}, t\right]$. The matrix function

$$
\begin{gathered}
P(t)=E\left[\left([z(t), x(t)]-\left[m_{z}(t), m_{x}(t)\right]\right) \times\right. \\
\left.\left([z(t), x(t)]-\left[m_{z}(t), m_{x}(t)\right]\right)^{T} \mid F_{t}^{Y}\right]
\end{gathered}
$$

is the estimation error variance matrix for this reformulated problem.

The obtained filtering system includes the two equations, (6) (or (7)) and (1), for the partially measured state $[z(t), x(t)]$ and the equation (8) for the observations $y(t)$, where $z(t)$ is a completely measured polynomial state with a polynomial multiplicative noise, $x(t)$ is an unmeasured polynomial state, and $y(t)$ is a linear observation process directly measuring the state $z(t)$. Applying the mean-square filter for incompletely measured polynomial states with a polynomial multiplicative noise over linear observations (see Basin and Maldonado (2011)) to the system (7),(1),(8) yields the desired filtering equations (4),(5). Finally, after representing the superior conditional moments of the system 
state as functions of the conditional expectation $m(t)$ and error variance $P(t)$ using the property of a Poisson random variable $x(t)-m(t)$ of representing the superior conditional moments of the system state as functions of the variance $P(t)$, (see Basin and Maldonado (2011) for details), a finite-dimensional system of the filtering equations, closed with respect to $m(t)$ and $P(t)$, can be obtained, if the initial condition $\left[z_{0}, x_{0}\right]$ for the extended state vector is conditionally Poisson.

Remark. Note that some particular cases of Theorem 1, like linear or bilinear systems with statedependent noises, were previously considered in Basin and Maldonado (2011), where the explicit mean-square finite-dimensional filtering equations were obtained. On the other hand, the general result of Theorem 1 allows one to design a suboptimal mean-square finitedimensional filter for any polynomial state confused with white Poisson noise disturbances over polynomial observations. Furthermore, since any nonlinear function can be approximated by a polynomial of a certain degree up to any precision, the result obtained in Theorem 1 would potentially lead to designing a suboptimal mean-square finite-dimensional filter for any nonlinear state with white Poisson noises over observations with a nonlinear drift.

In the following example, a closed form of the filtering equations will be obtained for a particular case of scalar second and third order polynomial functions $\rho(x, t), \sigma(x, t)$, and $h(x, t)$ in the equations (1) and (2). Nonetheless, application of the same procedure would result in designing a closed system of the filtering equations for any polynomial functions $\rho(x, t), \sigma(x, t)$, and $h(x, t)$ in $(1),(2)$.

\section{Example: Third Degree Sensor Filtering Problem for Quadratic System}

This section presents an example of designing the closed-form finite-dimensional filter for a quadratic state over third degree polynomial observations, where the initial condition for the extended state vector is assumed conditionally Poisson with respect to observations.

Let the unmeasured scalar state $x(t)$ satisfy the quadratic equation

$$
d x(t)=x^{2}(t) d t+d n_{1}(t), \quad x(0)=x_{0},
$$

and the observation process be given by the scalar third degree sensor equation

$$
d y(t)=x^{3}(t) d t+d n_{2}(t)
$$

where $n_{1}(t)$ and $n_{2}(t)$ are Poisson processes independent of each other and of a Poisson random variable $x_{0}$ serving as the initial condition in (9). The filtering problem is to find the mean-square estimate for the quadratic state (9), using the third degree sensor observations (10).

Let us reformulate the problem, introducing the stochastic process $z(t)=h(x, t)=x^{3}(t)$. Using the Ito formula (see Pugachev and Sinitsyn (2001)) for the stochastic differential of the cubic function $h(x, t)=$ $x^{3}(t)$, where $x(t)$ satisfies the equation (9), the following equation is obtained for $z(t)$

$d z(t)=\left(3 x(t)+3 x^{4}(t)\right) d t+3 x^{2}(t) d n_{1}(t), \quad z(0)=z_{0}$.

Taking into account that $z(t)=x^{3}(t)$, the last equation takes the form

$d z(t)=(3 x(t)(1+z(t))) d t+3 x^{2}(t) d n_{1}(t), \quad z(0)=z_{0}$.

Here, $\frac{\partial h(x, t)}{\partial x}=3 x^{2}(t), \quad \frac{1}{2} \frac{\partial h^{2}(x, t)}{\partial x^{2}}=3 x(t)$, and $\frac{\partial h(x, t)}{\partial t}=0$, given that $h(x, t)$ does not explicitly depend on $t$; therefore, $f(x, t)=3 x(t)+3 x^{4}(t)=$ $3 x(t)(1+z(t))$ and $g(x, t)=3 x^{2}(t)$. The initial condition $\left[z_{0}, x_{0}\right]$ is considered conditionally Poisson with respect to observations (see the paragraph preceding (7) for details). In terms of the process $z(t)$, the observation equation (10) takes the form

$$
d y(t)=z(t) d t+d n_{2}(t) .
$$

The obtained filtering system includes two equations, (11) and (9), for the partially measured state $[z(t), x(t)]$ and the equation (12) for the observations $y(t)$, where $z(t)$ is a completely measured second degree state with a multiplicative quadratic noise, $x(t)$ is an unmeasured quadratic state, and $y(t)$ is a linear observation process directly measuring the state $z(t)$. Hence, the designed mean-square filter can be applied for solving this problem. The filtering equations $(4),(5)$ take the following particular form for the system $(11),(9),(12)$

$$
\begin{gathered}
d m_{1}(t)=\left(3 m_{2}(t)+3 m_{1}(t) m_{2}(t)+3 P_{12}(t)\right) d t+(13) \\
P_{11}(t)\left[d y(t)-m_{1}(t) d t\right], \\
d m_{2}(t)=\left(m_{2}^{2}(t)+P_{22}(t)\right) d t+P_{12}(t)\left[d y(t)-m_{1}(t) d t\right],
\end{gathered}
$$

with the initial conditions $m_{1}(0)=E\left(z_{0} \mid y(0)\right)=m_{10}$ and $m_{2}(0)=E\left(x_{0} \mid y(0)\right)=m_{20}$,

$$
\begin{gathered}
\dot{P}_{11}(t)=12 P_{12}(t)+9 m_{2}^{4}(t)+9 P_{22}(t)+ \\
36 P_{22}(t) m_{2}(t)+54 P_{22}(t) m_{2}^{2}(t)+ \\
27 P_{22}^{2}(t)+12 P_{11}(t) m_{2}(t)-P_{11}^{2}(t),
\end{gathered}
$$




$$
\begin{gathered}
\dot{P}_{12}(t)=3 P_{12}(t)+7 P_{22}(t)+8 P_{12}(t) m_{2}(t)+ \\
3 m_{2}^{2}(t)-P_{11}(t) P_{12}(t), \\
\dot{P}_{22}(t)=1+2 P_{22}(t)+4 P_{22}(t) m_{2}(t)-P_{12}^{2}(t),
\end{gathered}
$$

with the initial condition $P(0)=E\left(\left(\left[z_{0}, x_{0}\right]^{T}-\right.\right.$ $\left.m(0))\left(\left[z_{0}, x_{0}\right]^{T}-m(0)\right)^{T} \mid y(0)\right)=P_{0}$. Here, $m_{1}(t)$ is the estimate for the state $z(t)=x^{3}(t)$ and $m_{2}(t)$ is the estimate for the state $x(t)$.

The estimates obtained upon solving the equations (13)-(17) are compared to the estimates satisfying the filtering equations designed for systems with white Gaussian noises for the quadratic state (11) over the third order polynomial observations (10), which are obtained in Basin et al. (2010):

$$
\begin{gathered}
d m_{G 1}(t)=\left(3 m_{G 2}(t)+3 m_{G 1}(t) m_{G 2}(t)+\right. \\
\left.3 P_{G 12}(t)\right) d t+P_{G 11}(t)\left[d y(t)-m_{G 1}(t) d t\right], \\
d m_{G 2}(t)=\left(m_{G 2}^{2}(t)+P_{G 22}(t)\right) d t+ \\
P_{G 12}(t)\left[d y(t)-m_{G 1}(t) d t\right],
\end{gathered}
$$

with the initial conditions $m_{G 1}(0)=E\left(z_{0} \mid y(0)\right)=$ $m_{G 10}$ and $m_{G 2}(0)=E\left(x_{0} \mid y(0)\right)=m_{G 20}$,

$$
\begin{gathered}
\dot{P}_{G 11}(t)=6 P_{G 12}(t)+9 m_{G 2}^{4}(t)+9 P_{G 22}(t)+ \\
36 P_{G 22}(t) m_{G 2}(t)+54 P_{G 22}(t) m_{G 2}^{2}(t)+ \\
27 P_{G 22}^{2}(t)+12 P_{G 11}(t) m_{G 2}(t)-P_{G 11}^{2}(t), \\
\dot{P}_{G 12}(t)=6 P_{G 22}(t)+8 P_{G 12}(t) m_{G 2}(t)+3 m_{G 2}^{2}(t) \\
-P_{G 11}(t) P_{G 12}(t), \\
\dot{P}_{G 22}(t)=1+4 P_{G 22}(t) m_{G 2}(t)-P_{G 12}^{2}(t),
\end{gathered}
$$

with the initial condition $P_{G}(0)=E\left(\left(\left[z_{0}, x_{0}\right]^{T}-\right.\right.$ $\left.\left.m_{G}(0)\right)\left(\left[z_{0}, x_{0}\right]^{T}-m_{G}(0)\right)^{T} \mid y(0)\right)=P_{G 0}$.

Numerical simulation results are obtained solving the systems of filtering equations (13)-(17) and (18)(22). The obtained values of the state estimates $m_{2}(t)$, satisfying the equation (14), and $m_{G 2}(t)$, satisfying the equation (19), are compared to the real values of the state variable $x(t)$ in $(9)$.

For the filters (13)-(17), (18)-(22) and the reference system (11),(9),(12) involved in simulation, the following initial values are assigned: $x_{0}=0, z_{0}=18, m_{10}=$ $m_{G 10}=1000, m_{20}=m_{G 20}=10, P_{11}(0)=P_{G 11}(0)=$ $15, P_{12}(0)=P_{G 12}(0)=3, P_{22}(0)=P_{G 22}(0)=1$. Poisson disturbances $d n_{1}(t)$ and $d n_{2}(t)$ are generated using the Simulink chart suggested in Basin et al. (2007). Note that the noise power can be changed varying the terms $\sigma(x, t)$ and $B(t)$ in (1),(2), which would lead to changing the corresponding terms in the filtering equations (4),(5). The simulation interval is set to $[0,4.0575]$, since the estimation error given by the filter designed for systems with white Gaussian noises diverges to infinity at this time.
Figure 1 shows the graphs of the errors between the reference state $x(t)(9)$ and its estimate $m_{2}(t)$ (14), and the reference state $z(t)=x^{3}(t)(11)$ and its estimate $m_{1}(t)(13)$, in the entire simulation interval from $t_{0}=0$ to $T=4.0575$. It can be observed that the estimation errors converge to the real states very rapidly and then maintain zero mean value, in spite of a considerable error in the initial conditions, $m_{20}-x_{0}=10, m_{10}-z_{0}=982$. The estimation error for the state $x(t)$ at $T=4.0575$ is equal to $m_{2}(4.0575)-x(4.0575)=0.1646$. Figure 2 shows the graph of the errors between the reference state $x(t)$ (9) and the estimate $m_{G 2}(t)(19)$, and the reference state $z(t)=x^{3}(t)(11)$ and its estimate $m_{G 1}(t)(18)$, in the entire simulation interval from $t_{0}=0$ to $T=4.0575$. Note that although the estimate equations (13)-(14) coincide with the estimate equations (18)-(19), the designed filter for systems with white Poisson noises shows very good overall performance at every time moment $t$ of the simulation interval (Fig. 1), whereas the estimation error of the estimate $m_{G 2}(t)$ provided by the filter available for systems with white Gaussian noises diverges to infinity at $T=4.0575$ (Fig. 2).

Thus, it can be concluded that the obtained filter (13)-(17) solves the third order sensor filtering problem for the system (9),(10), where the state and observations are polynomials corrupted with white Poisson noises, and yields a reliable estimate of the unmeasured state.

\section{Conclusions}

This paper presents an approximate finite-dimensional filter for polynomial system states confused with white Poisson noises over polynomial observations. It is shown that the proposed finite-dimensional filter can be obtained in a closed form for any polynomial functions in state and observation equations. In the example, the closed-form solution is obtained to the filtering problem for a quadratic state over third degree polynomial observations, assuming a conditionally Poisson initial condition for the extended third order state vector. The resulting filter yields a reliable and rapidly converging estimate, in spite of a significant difference in the initial conditions between the state and estimate, whereas the filter designed for systems with white Gaussian noises behaves unsatisfactorily. Although this conclusion follows from the developed theory, the numerical simulation serves as a convincing illustration. 

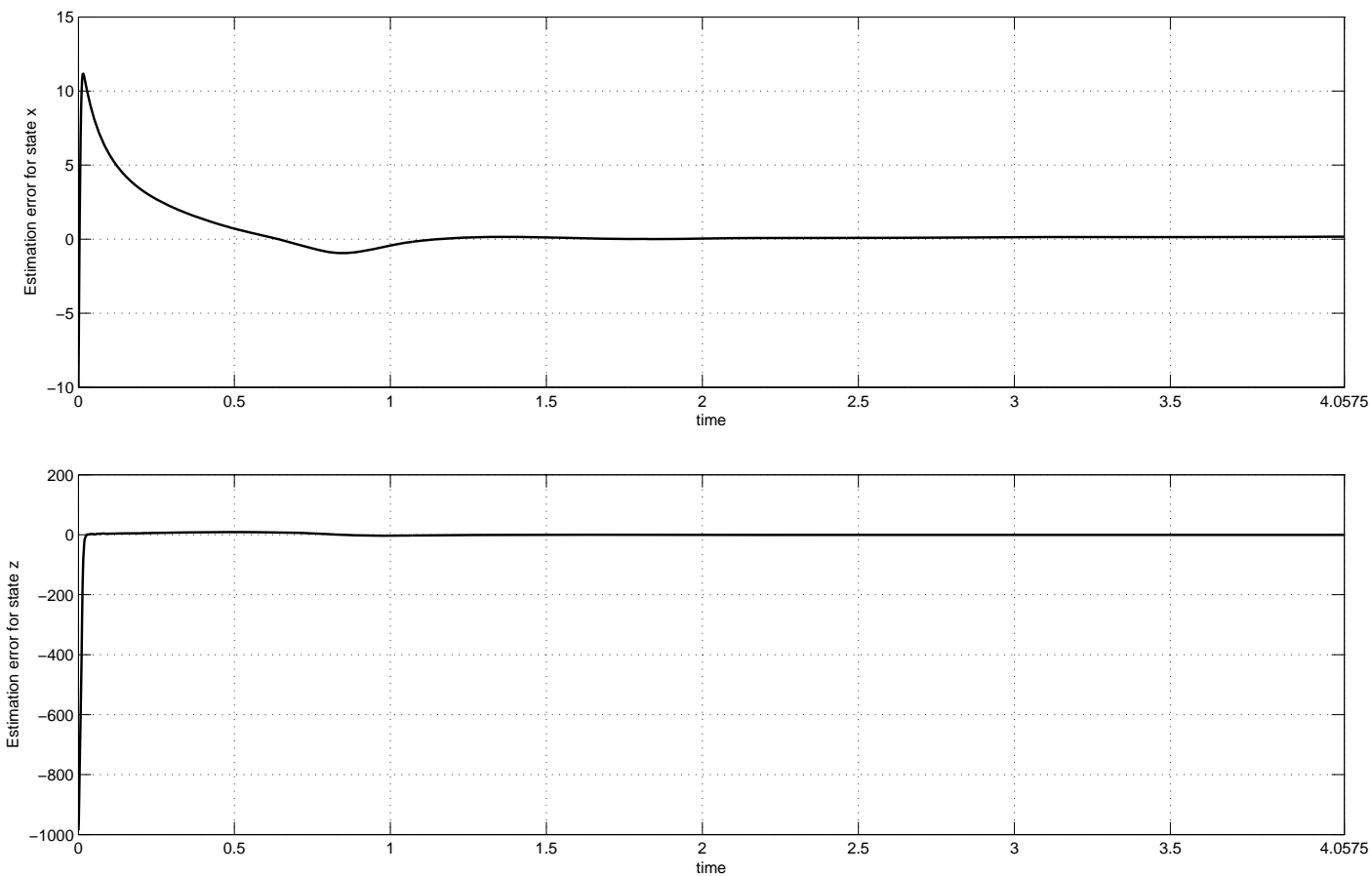

Figure 1: Top. Graph of the estimation error between the reference state $x(t)(9)$ and its estimate $m_{2}(t)(14)$ in the interval $[0,4.0575]$. Bottom. Graph of the estimation error between the reference state $z(t)$ (11) and its estimate $m_{1}(t)(13)$ in the interval [0, 4.0575].
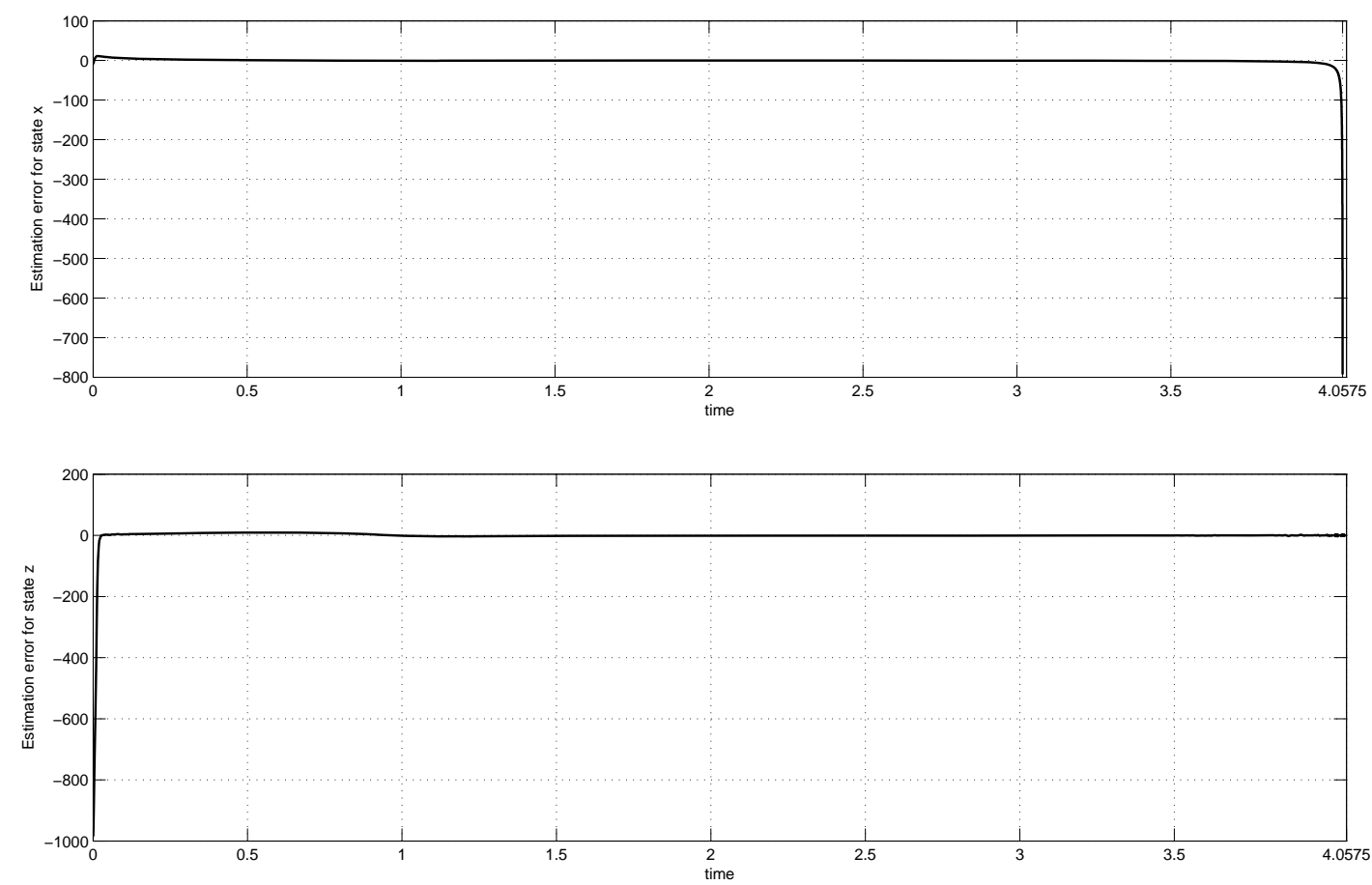

Figure 2: Top. Graph of the estimation error between the reference state $x(t)(9)$ and the estimate $m_{G 2}(t)(19)$ in the interval $[0,4.0575]$. Bottom. Graph of the estimation error between the reference state $z(t)$ (11) and the estimate $m_{G 2}(t)(18)$ in the interval [0,4.0575]. 


\section{References}

Basin, M. New Trends in Optimal Filtering and Control for Polynomial and Time-Delay Systems. Berlin: Springer, 2008.

Basin, M., Alcorta-Garcia, M., and Pena, D. Simulation and optimal filtering for linear systems with poisson white noises. Dynamics of Continuous, Discrete and Impulsive Systems. Series B, 2007. 14:221231.

Basin, M., Calderon-Alvarez, D., and Skliar, M. Optimal filtering for incompletely measured polynomial states over linear observations. International J. Adaptive Control and Signal Processing, 2008. 22:482-494.

Basin, M. and Maldonado, J. Mean-square filter design for nonlinear polynomial systems with poisson noise. Proc. 2011 American Control Conference, 2011. page Ref. no. WeB02.1.

Basin, M., Shi, P., and Calderon-Alvarez, D. Optimal filtering for incompletely measured polynomial systems with multiplicative noises. Circuits, Systems and Signal Processing, 2009. 28:223-239. doi:10.1007/s00034-008-9083-2.

Basin, M., Shi, P., and Calderon-Alvarez, D. Approximate finite-dimensional filtering for polynomial states over polynomial observations. International Journal of Control, 2010. 83:724-730.

Benes, V. Exact finite-dimensional filters for certain diffusions with nonlinear drift. Stochastics, 1981. 5:65-92. doi:10.1080/17442508108833174.

Dupé, F., Fadili, M., and Starck, J.-L. Image deconvolution under poisson noise using sparse representations and proximal thresholding iteration. Proc. IEEE ICASSP - Int. Conf. on Acoustics, Speech, and Signal Processing, 2008. pages 761-764.

Fleming, W. and McEneaney, W. Robust limits of risk sensitive nonlinear filters. Mathematics of Control, Signals and Systems, 2001. 14:109-142. doi:10.1007/PL00009879.

Fridman, E. and Shaked, U. On regional nonlinear $\mathrm{H}_{\infty}$ filtering. Systems and Control Letters, 1997. 29:233240. doi:10.1016/S0167-6911(96)00061-8.

Gao, H. and Chen, T. $\mathrm{H}_{\infty}$ estimation for uncertain systems with limited communication capacity. IEEE Transactions on Automatic Control, 2007. 52:20702084. doi:10.1109/TAC.2007.908316.
Gao, H., L., J. L., Xie, and Wang, C. New approach to mixed $\mathrm{H}_{2} / \mathrm{H}_{\infty}$-filtering for polytopic discrete-time systems. IEEE Transactions on Signal Processing, 2005. 53:3183-3192.

Gao, H. and Wang, C. A delay-dependent approach to robust $\mathrm{H}_{\infty}$ filtering for uncertain discrete-time state-delayed systems. IEEE Transactions on Signal Processing, 2004. 52:1631-1640.

Hannequin, P. and Mas, J. Statistical and heuristic image noise extraction (shine): A new method for processing poisson noise in scintigraphic images. Phys. Med. Biol., 2002. 47:4329-4344. doi:10.1088/0031$9155 / 47 / 24 / 302$.

Hazewinkel, M., Marcus, S., and Sussmann, H. Nonexistence of exact finite-dimensional filters for conditional statistics of the cubic sensor problem. Systems and Control Letters, 1983. 5:331-340.

Kalman, R. and Bucy, R. New results in linear filtering and prediction theory. ASME Trans., Part D (J. of Basic Engineering), 1961. 83:95-108.

Kolmanovsky, I. and Maizenberg, T. Optimal containment control for a class of stochastic systems perturbed by poisson and wiener processes. Proc. 2002 American Control Conf., 2002a. pages 322-327.

Kolmanovsky, I. and Maizenberg, T. Optimal containment control for a class of stochastic systems perturbed by poisson and wiener processes. IEEE Trans. on Automatic Control, 2002b. 47:1641-1645.

Kushner, H. On differential equations satisfied by conditional probability densities of markov processes. SIAM J. Control, 1964. 12:106-119.

Lu, H., Liang, Z., and Chen, D. A combined transformation of ordering spect sinograms for signal extraction from measurements with poisson noise. SPIE Proc., 2001. 4322:1431-1438.

Mahmoud, M. and Shi, P. Robust kalman filtering for continuous time-lag systems with markovian jump parameters. Automatica, 2003. 50:98-105.

Nguang, S. and Fu, M. Robust nonlinear $\mathrm{H}_{\infty}$ filtering. International Journal of Robust and Nonlinear Control, 1996. 32:1195-1199. doi:10.1016/00051098(96)00067-2.

Oksendal, B. Stochastic Differential Equations. Berlin: Springer, 2006.

Pugachev, V. Probability Theory and Mathematical Statistics for Engineers. London, Pergamon, 1984. 
Pugachev, V. and Sinitsyn, I. Stochastic Systems: Theory and Applications. Singapore: World Scientific, 2001.

Shen, B., Wang, Z., Shu, H., and Wei, G. $\mathrm{H}_{\infty}$ filtering for nonlinear discrete-time stochastic systems with randomly varying sensor delays. Automatica, 2009. 45:1032-1037. doi:10.1016/j.automatica.2008.11.009.

Shi, P. Filtering on sampled-data systems with parametric uncertainty. IEEE Transactions on Automatic Control, 1998. 43:1022-1027.

Tucker, H. A Graduate Course in Probability. New York: Academic Press, 1967.

Wang, Z., Ho, D., Liu, Y., and Liu, X. Robust $\mathrm{H}_{\infty}$ infinity control for a class of nonlinear discrete time-delay stochastic systems with missing measurements. Automatica, 2009. 45:684-691. doi:10.1016/j.automatica.2008.10.025.

Wang, Z., Lam, J., and Liu, X. Nonlinear filtering for state delayed systems with markovian switching. IEEE Transactions on Signal Processing, 2003. 51:2321-2328. doi:doi:10.1109/TSP.2003.815373.

Wang, Z., Liu, Y., and Liu, X. $\mathrm{H}_{\infty}$ filtering for uncertain stochastic time-delay systems with sectorbounded nonlinearities. Automatica, 2008. 44:12681277. doi:10.1016/j.automatica.2007.09.016.

Wei, G., Wang, Z., and Shu, H. Robust filtering with stochastic nonlinearities and multiple missing measurements. Automatica, 2009. 45:836-841. doi:10.1016/j.automatica.2008.10.028.

Wonham, W. Some applications of stochastic differential equations to nonlinear filtering. SIAM J. Control, 1965. 2:347-369.

Xie, L., Souza, C. D., and Wang, Y. Robust filtering for a class of discrete-time uncertain nonlinear systems. International Journal of Robust and Nonlinear Control, 1996. 6:297-312. doi:10.1002/(SICI)10991239(199605)6:4;297::AID-RNC234¿3.0.CO;2-V.

$\mathrm{Xu}$, S. and Chen, T. Robust $\mathrm{H}_{\infty}$ filtering for uncertain impulsive stochastic systems under sampled measurements. Automatica, 2003. 39:509-516. doi:10.1016/S0005-1098(02)00248-0.

$\mathrm{Xu}$, S. and van Dooren, P. Robust $\mathrm{H}_{\infty}$ filtering for a class of nonlinear systems with state delay and parameter uncertainty. Int. J. Control, 2002. 75:766774. doi:10.1080/00207170210141815.
$\mathrm{Xu}, \mathrm{S} ., \mathrm{Lam}$, J., Gao, H., and Zhou, Y. Robust $\mathrm{H}_{\infty}$ filtering for uncertain discrete stochastic systems with time delays. Circuits, Systems and Signal Processing, 2005. 24:753-770. doi:10.1007/s00034-005-09211.

Yau, S. S.-T. Finite-dimensional filters with nonlinear drift i: a class of filters including both kalman-bucy and benes filters. J. Math. Systems, Estimation, and Control, 1994. 4:181-203.

Yaz, E. and Yaz, Y. State estimation of uncertain nonlinear systems with general criteria. Applied Mathematics Letters, 2001. 14:605-610. doi:10.1016/S0893-9659(00)00201-9.

Zhang, B., Fadili, M., Starck, J., and Dige, S. Fast poisson noise removal by biorthogonal haar domain hypothesis testing. Statistical Methodology, 2008a. 5:387-396. doi:10.1016/j.stamet.2008.02.004.

Zhang, B., Fadili, M., and Starck, J.-L. Wavelets, ridgelets, and curvelets for poisson noise removal. IEEE Trans. on Image Processing, 2008b. 17:10931108. doi:10.1109/TIP.2008.924386.

Zhang, H., Basin, M., and Skliar, M. Ito-volterra optimal state estimation with continuous, multirate, randomly sampled, and delayed measurements. IEEE Transactions on Automatic Control, 2007. 52:401416. doi:10.1109/TAC.2007.892383.

Zhang, W., Chen, B., and Tseng, C. Robust $\mathrm{H}_{\infty}$ filtering for nonlinear stochastic systems. IEEE Transactions on Signal Processing, 2005. 53:589-598. doi:10.1109/TSP.2004.840724. 